\title{
Review of methods for researching multistrata systems
}

Richard Coe and Betha Lusiana

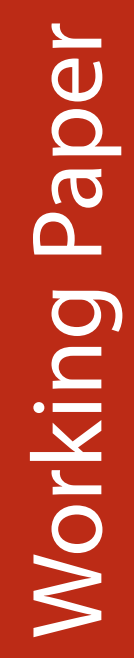




\section{Review of methods for researching multistrata systems}

Coe Richard ${ }^{1}$ and Lusiana Betha ${ }^{2}$

${ }^{1}$ World Agroforestry Centre (ICRAF), Nairobi, Kenya.

${ }^{2}$ World Agroforestry Centre, South East Asia, Bogor, Indonesia.

\section{Correspondence:}

Email: r.coe@cgiar.org

Phone: +254 (0)20 7224326

PO Box 30677, Nairobi 00100, Kenya 


\begin{abstract}
In the introduction to the special journal issue resulting from the First Symposium on Multistrata Agroforestry Systems (1999), Muschler and Beer (2001) point out that traditional approaches to studying multistrata systems with perennial crops tend to over simplify them. They identified new questions for research focusing on complexity and processes interacting at multiple scales, and conclude that 'Undoubtedly these new questions require innovative methods appropriate at different scales, including modelling approaches to cope with complexity at several scales simultaneously'. Have innovative methods been developed and proved useful? We review the literature from the 7 years since that symposium to identify and describe (a) trends in methods for researching multistrata systems (b) new research methods that have been successfully used (c) emerging methods challenges. Results suggest that there has been little innovation in research methods in this area in the last 7 years. Most of the questions being addressed are not specific to multistrata systems and use methods common in other agricultural research. There seems to be little research on complexity at several scales, but it is not clear whether this is due to methodological limitations.
\end{abstract}




\section{Introduction}

The understanding of Multi-Strata Agroforestry Systems (MSAS) has been recognised as posing challenges to research methods. In their introduction to the special journal edition resulting from the first conference on MSAS in 1999, Muschler and Beer (2001) summarised the research challenges on MSAS as being in the areas of (a) understanding the vegetation structures that lead to sustainability of MSAS (b) understanding simplifications that can be made to complex MSAS without losing sustainability (c) understanding the market and policy environments that lead to continued use of MSAS and (d) understanding how MSAS contribute to ecosystem services. All these research areas require working with the complexity in MSAS. The complexity arises from the many interacting components, sometimes taken as a definition of a complex system. As a landuse, MSAS are challenging to study due to their variety, their complex evolution or 'cropping cycle' and the heterogeneous landscape mosaics in which they are found, among other characteristics.

Muschler and Beer (2001) suggested that new research methods are needed to tackle the important questions surrounding MSAS, and highlighted methods that:

1. Use process-based models.

2. Work at multiple scales.

3. Integrate biophysical, social and economic aspects.

Our aim in this paper is to assess the extent to which new methods have been developed and used in MSAS, particularly methods that encompass these characteristics. Have they provided new insights? Are there other methods that are proving useful? Have new methods challenges become apparent?

\section{Approach}

Our approach has been to review the methods described in papers published since 2000 reporting research on MSAS in international refereed journals. It has not been possible to do a comprehensive or exhaustive review. That would have been too large a task, and anyway impossible as the domain (MSAS) is not well enough defined. Instead we sought a range of papers reporting research relevant to the current conference (i.e. falling into one of the four conference themes). The results are therefore qualitative and indicative, rather than quantitative. Fifty-six papers were specifically reviewed for this study. Twenty-two of these report research from South East Asia, 16 from Central America, 6 from South America. The remainder come from other regions or are not regionally specific. Over half the papers focused on a specific commodity, dominated by coffee $(22 / 56)$. We recognise that this is not a large sample of papers for such a multifaceted research area but hope it is sufficient to reveal new important methods and outstanding problems.

For each paper we attempted to evaluate the methods used, looking for novelty and for notably strong or weak points. We specifically looked for papers that use methods highlighted by Muschler and Beer (2001) - modelling, multiscale and integrating. Note that our sources are studies that have been published in the international peer reviewed literature. Hence, by the usual standards, the methods are acceptable and there is something novel in the results. Further, as every study has something unique about it, at some level the details of methods are likely to include something novel. However, we were searching for methodological advances that appear substantial, allowing new avenues of research, rather than simply adapting a well-known method to a specific situation.

Note that the whole of this paper focuses on the methods not the results of the research. 


\section{Comparisons and experiments}

A majority (31/56) of the papers involve comparison of one or more MSAS with another landuse or uses, including simpler tree or crop systems, plantations or 'natural' forest. In none of these was a designed experiment used, with alternative systems randomly allocated to plots as treatments, though some used data from previously published experiments. Although randomised experiments have been successfully used in system comparison research on MSAS - see the review by Somarriba et al. (2001) - the reasons for not making more use of them are clear. They include:

- Systems take long to mature so experiments are long term investments.

- Experiments require researchers to take decisions on exact specification of an endless variety of MSAS to be investigated.

- They require large plots and costly maintenance.

- It may be unfeasible to include some systems, particularly natural forest, as randomised treatments.

- They can only answer questions at the spatial scale of the experimental plot or smaller.

- They simplify or eliminate some of the interactions and processes.

However, the alternative of making inferences from observational studies can also be difficult. The problems are well documented and include confounding, the difficulty of inferring causation from correlation, and 'spurious correlation' induced by unmeasured variables. Two designs common in work on MSAS highlight the problems. 'Gradsects' are sampling transects taken along the gradient of an environmental variable (Gillison et al., 2004). Conclusions concerning the impact of that variable depend on there not being others that vary systematically in the same way. This can be confirmed for any such variable that is hypothesised, and hence measured, but not for one that does not occur to the researcher. A chronosequence is a series of plots representing different ages of a system, for example times since conversion from natural forest. These are examined to make inferences about the evolution of systems in time, yet suffer from the usual constraints of drawing conclusions about change in time from cross-sectional data. Strong assumptions are needed about what stays constant. These difficulties are rarely explicitly and critically examined in the papers we reviewed.

Randomised experiments continue to be used to investigate components and component processes in MSAS. Examples include studies of fire (Ketterings et al., 2002), decomposition (Kurtzatkowski et al., 2004) and gap planting (Siebert, 2000). These methods are not unique to MSAS.

\section{Spatial scale}

Plots in both experiments and observational studies of MSAS are typically of $10^{2}$ to $10^{3}$ $\mathrm{m}^{2}$. Important properties of MSAS, such as sustainability, maintenance of biodiversity and hydrological functions, are only apparent at larger spatial scales. Exactly what scales are important are among the outstanding research questions, but are often assumed to be a vague 'landscape scale', maybe $10^{6}$ to $10^{8} \mathrm{~m}^{2}$. Muschler and Beer (2001) summarised the need to have methods to work at these larger scales, and to link between scales. However the large majority of the papers reviewed collect empirical data only at the plot scale, with corresponding social and economic data collected at household level. Again the reasons for this are clear as alternatives are not straightforward. 
There are two options for studying processes at larger scale:

1. Collect empirical data at those larger scales. This is rarely possible, as most measurement tools can only practically be applied at plot (and smaller) scale. There are exceptions, such as net runoff that can be measured in catchments, but these are rare.

2. Collect data at smaller scales and 'scale up'.

Scaling measurements to larger areas is often not straightforward. We know, for example, that the total crop production of a landscape is the area multiplied by the average production per plot. However the same is not true of run-off or erosion. Scaling up of such variables requires either development of empirical rules or modelling the processes. In the case of runoff, the difficulty arises through lateral flows and sinks, and a spatially explicit process model of the landscape is needed.

Measurements of biodiversity, such as species richness, are known not to scale in a simple way - the total species count in a landscape is not the sum of the species counts in each sample plot. Tools for handling this, such as rarefaction and species cumulation curves are well known, and have been used in several of the biodiversity papers in our sample. However, they cannot be used uncritically and it is not always obvious what the results represent. For example, consider a landscape that is a mosaic of MSAS and other land uses, including forest. If a sample of plots were taken in such a landscape, and separate species cumulation curves calculated for forest and MSAS plots, what do these represent? They do not represent the biodiversity of a landscape with just one of those components, nor do they measure the contribution of each component to the overall landscape diversity.

\section{Integrating social, economic and biophysical aspects}

Some progress does appear to have been made here, with studies of the social, policy and economic drivers of adoption and continued management of MSAS (for example Suyanto and Otsuka (2001)). However these have not used methods specific to MSAS. The approaches do not appear to be novel. Likewise, analyses of financial and economic aspects of MSAS use methods applied in analysis of other agricultural enterprises, though some details may be adapted for MSAS. For example, Guo et al. (2006) used the Land Expectation Value (LEV) as a criterion for evaluating alternative MSAS. This measure had previously been used in forest economics for analysis of a single rotation. Guo et al. adapted it for MSAS that have evolving cost and benefit streams, assuming an infinite repetition of the same rotations.

\section{Modelling processes}

Remarkably few papers in our survey developed process-based models of MSAS that give the insights into complexity demanded by Muschler and Beer (2001). Some of the potential is illustrated by Mialet-Serra (2001) and Harja and Vincent (2006), both of which use models of canopy structure and tree architecture to understand the light regime in MSAS. Such models could be used to derive management guidelines (e.g. pruning, thinning and gap planting).

One example of a process model stands out in the way it generates cross-scale insights and integrates biophysical and socioeconomic aspects. van Noordwijk (2002) introduces the FALLOW model. The model describes evolution of a landscape of 100 heterogeneous 
fields managed by farmers. Farmers take decisions on which fields to crop and which to leave fallow, based on their current fertility, crop needs and returns. When fields are fallowed, their biodiversity and carbon stocks evolve. When fields are cropped their fertility declines. The landscape level values of these can thus be described. In the default form the model describes a generic landscape, with results that depend on the variability between plots and the interactions between them, rather than on an average plot. This type of model could be elaborated in any number of ways to describe further processes and further influences on farmer decision making. Note that this style of modelling does not aim to simulate a specific landscape, making predictions for an identified geographical location. Its aim is to reveal possible and likely patterns such as the effect of policy changes or simple scaling rules for field data. Most importantly, it provides a means for deriving landscape level implications of plot level dynamics.

\section{Limitations with current methods}

If few new research methods for MSAS are emerging, are the current methods effective? Within our sample of studies, methods are clearly giving results or they would not have been published. However there are common limitations of the methods used. Most notable are:

- Sample sizes are often very small, with little spatial or temporal variability sampled.

- Many designs are hierarchical, for example with plots sampled within 'landscapes' and landscapes sampled within 'sites'. The important comparisons are often at a high level in this hierarchy - for example between sites with and without MSAS. Yet at this level there is little or no replication.

- The difficulties in interpretation of observational studies noted above.

- Use of over-simplistic statistical analysis tools, with old favourites used without critical evaluation of their relevance or validity.

However, there is nothing special about MSAS in these respects. Review of research reports in any area of agriculture, landuse and ecology will show similar problems, and they can often be traced to trying to do something with very limited resources.

\section{Are MSAS special?}

Judged by the volume of publication, there has been much progress in research on MSAS in the last 7 years. Our brief survey suggests that most of the issues being studied are not unique to MSAS and therefore the research methods employed are not unique either. At some level of discussion this will always be true, such as if we classify empirical research methods as either experimental or observational. However, there are questions that seem to be peculiar to MSAS - for example, those on complexity summarised by Muschler and Beer (2001) - and for which at least adaptations of research methods are needed. Is the failure to address these problems one of methods or are there other reasons? Our approach based on published results cannot answer this, as researchers rarely publish explanations of the questions they did not investigate because they were stuck with methods. Our experience suggests there are other reasons. 
If those questions about the complexity of MSAS are not being addressed the reasons could be:

1. They are not considered important by researchers.

2. They are important, but there are methodology constraints.

3. They are important to researchers but not to funders of research.

The first of these will, we hope, be evaluated during this conference. The second is unlikely to be the case. Researchers have always been innovative with methods when needed, and we have found some developments. It can take a long time for a new research method to become a standard tool, widely understood, used and accepted. But within the research world of MSAS, we would expect some useful innovations to be adopted and developed rapidly. The third option is very real and imposes many limitations to research. Trends in research are often set outside the research community. As an example, the rapid increase in MSAS papers on coffee and biodiversity are the result of growing interest in these topics among consumers and conservationists. With much research in tropical land use being financed by development assistance funding, researchers are often constrained to do things that are (relatively) quick and easy and have apparent immediate application. This situation is not conducive to sustained effort at understanding more fundamental questions.

We are not going to see a quick change in this situation. However there are two things that can be done:

1. Research institutions (including informal institutes of networks of scientists) can keep a focus on the questions they consider fundamental, and make sure that each piece of development-related research adds to that fundamental agenda. This is in contrast to the alternative, in which development-related research funding is accepted in an opportunistic way without a sustained effort in any direction.

2. Scientists and their institutions can actively promote uptake of newer methods, for example by making them available in accessible forms, and providing good guidelines, documentation and training opportunities.

\section{Conclusions}

A brief review of recent research literature on multistrata agroforestry systems suggests that there have been few methodological innovations. Progress in understanding some aspects of these systems is being made using methods common across much agricultural research. However, there are some questions, particularly focusing on complexity, which are not being addressed. Methods may not be readily available for these, but will probably be developed when researchers are encouraged to focus on them. 


\section{References}

Gillison AN, Liswanti N, Budidarsono S, Noordwijk M, Tomich TP. 2004 Impact of cropping methods on biodiversity in coffee agroecosystems in Sumatra, Indonesia. Ecology and Society 9(2): 7

Guo Z, Zhang Y, Deegen P, Uibrig H. 2006. Economic analyses of rubber and tea plantations and rubber-tea intercropping in Hainan, China. Agroforestry Systems 66: 117-127

Harja D, Vincent G. 2006. The SEXI-FS Spatially explicit individual-tree-based agroforest simulator. In: van Noordwijk $\mathrm{M}$ and $\mathrm{O}^{\prime}$ Connor T (Eds.) Highlights of ongoing research of the World Agroforestry Centre in Indonesia. ICRAF Southeast Asia Working Paper 2006-1, pp 4-7

Ketterings QM, van Noordwijk M, Bigham JM. 2002. Soil phosphorus availability after slashand-burn fires of different intensities in rubber agroforests in Sumatra, Indonesia. Agriculture, Ecosystems and Environment 92:37-48

Kurtzatkowski D, Martius C, Höfer H, Garcia M, Förster B, Beck L and Vlek P. 2004. Litter decomposition, microbial biomass and activity of soil organisms in three agroforestry sites in central Amazonia. Nutrient Cycling in Agroecosystems 69:257-267

Mialet-Serra I, Dauzat J, Auclair D. 2001. Using plant architectural models for estimation of radiation transfer in a coconut based agroforestry systems. Agroforestry Systems 53:141149

Muschler R, Beer J. 2001. Multistrata agroforestry systems with perennial crops. Agroforestry Systems 53: v-vi

Siebert SF. 2000. Survival and growth of rattan intercroppped with coffee and cacao in agroforests of indonesia. Agroforestry Systems 50:95-102

Somarriba E, Beer J, Muschler R. 2001. Research methods for multistrata agroforestry systems with coffee and cacao: recommendations from two decades of research at CATIE. Agroforestry Systems 53: 195-203

Suyanto S, Otsuka K 2001. From deforestation to development of agroforests in customary land tenure areas of Sumatra. Asian Economic Journal 15:1-17

van Noordwijk M 2002. Scaling trade-offs between crop productivity, carbon stocks and biodiversity in shifting cultivation mosaics: the FALLOW model. Ecological Modelling 149:113-126 


\section{About us}

The World Agroforestry Centre is part of the alliance of the Consultative Group on International Agricultural Research (CGIAR) centres dedicated to generating and applying the best available knowledge to stimulate agricultural growth, raise farmers' incomes, and protect the environment.

\section{Our Vision}

The Centre's vision is a rural transformation in the developing world as smallholder households strategically increase their use of trees in agricultural landscapes to improve their food security, nutrition, income, health, shelter, energy resources and environmental sustainability. This vision is founded on the growing role of trees in sustaining livelihoods and agroecosystems, the Centre's experience and comparative advantage in advancing agroforestry research for development, and global commitment to achieving the Millennium Development Goals.

\section{Our Mission}

The Centre's mission is to generate science-based knowledge about the diverse roles that trees play in agricultural landscapes, and use its research to advance policies and practices that benefit the poor and the environment.

The Centre is guided by the broad development challenges pursued by the CGIAR. These include poverty alleviation that entails enhanced food security and health, improved productivity with lower environmental and social costs, and resilience in the face of climate change and other external shocks.

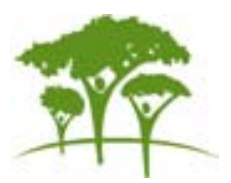

World Agroforestry Centre (ICRAF)

United Nations Ave, PO Box 30677-00100 Nairobi, Kenya

Tel: +25420722 4000 or via USA +16508336645

Fax: +25420722 4001 or via USA 16508336646

Email: icraf@cgiar.org

www.worldagroforestry.org 